\title{
Clinical features, management, and outcome of iliopsoas abscess associated with cardiovascular disorders: a hospital- based observational case series study
}

Sung-Yuan Hu ${ }^{1,2,3,4,5,6,7^{*}}$, Ming-Shun Hsieh ${ }^{7,8,9,10}$, Yao-Tien Chang ${ }^{1,4}$, Chih-Che Huang ${ }^{1,11}$, Che-An Tsai ${ }^{12}$, Chung-Lin Tsai ${ }^{13}$, Chiann-Yi Hsu ${ }^{11}$, Chia-Hui Shen ${ }^{11}$ and Yan-Zin Chang ${ }^{1,2,14^{*}}$

\begin{abstract}
Background: lliopsoas abscess (IPA) is a rare clinical entity and is difficult to diagnose due to its insidious onset and nonspecific symptoms. The association between IPA and cardiovascular disorders (CVD) has been rarely reported. Computed tomographic (CT) scan can provide a definitive diagnosis of IPA and associated foci of adjacent structures. IPA is a life-threatening condition, especially when associated with CVD.

Materials and methods: We conducted a hospital-based observational study of IPA associated with CVD. Data were collected from the electronic clinical database of Taichung Veterans General Hospital (1520-bed tertiary referral hospital in central Taiwan) between July 2007 and December 2017. The diagnosis of IPA associated with CVD was confirmed by classical findings on $C T$ and transesophageal echocardiography with compatible clinical presentation and cultures from pus/tissue and blood.

Results: Fifteen patients of IPA associated with CVD were studied. They included 12 males (80\%) and 3 females (20\%), with a mean age 63.2 \pm 16.9 years (31-85 years). CVD included stent-graft/endograft infection of abdominal aortic aneurysm (AAA) (40\%), primary mycotic AAA (33.3\%), and infective endocarditis (26.7\%). Staphylococcus aureus is the most common microorganism in pus/tissue cultures $(n=3,37.5 \%)$ and in blood cultures $(n=6,40 \%)$. The average length of hospital stay was $33.1 \pm 20.5$ days (range, 3-81 days; median, 33 days). Hospital stay lasted $42.6 \pm$ 19.2 days in the survival group and $19.0 \pm 14.1$ days $(P=0.018)$ in the non-survival group. Incidence of patients staying in the intensive care unit (ICU) with intubation $>3$ days was $33 \%$ in the survival group and $100 \%(P=0.028)$ in the non-survival group. Intra-hospital mortality rate was 40\%. Poor prognostic factors in the non-survival group were hypoalbuminemia, hyponatremia, involved disc/vertebral body and/or epidural abscess, and ICU stay with intubation $>3$ days. Cumulative survival rate was 25\% under conservative treatments and $66.3 \%$ under aggressive treatments $(P=0.038)$.

Conclusion: Due to high mortality rates, clinicians should keep a high suspicion index for IPA associated with CVD through clinical presentation, physical examination, and imaging study. Timely empiric antibiotics for common bacteria, drainage for IPA, endovascular repair, or vascular reconstruction by graft replacement or bypass with intensive care should be mandatory to shorten the hospital stay, reduce medical costs, and lower mortality rate.
\end{abstract}

Keywords: Computed tomography (CT), Stent-graft/Endograft infection, Infective endocarditis, lliopsoas abscess (IPA), Mycotic aneurysm

\footnotetext{
*Correspondence: song9168@pie.com.tw; yzc@csmu.edu.tw

${ }^{1}$ Institute of Medicine, Chung Shan Medical University, No. 110, Sec. 1,

Jianguo N. Rd, Taichung City 40201, Taiwan

Full list of author information is available at the end of the article
}

(c) The Author(s). 2019 Open Access This article is distributed under the terms of the Creative Commons Attribution 4.0 International License (http://creativecommons.org/licenses/by/4.0/) which permits unrestricted use, distribution, and reproduction in any medium, provided you give appropriate credit to the original author(s) and the source, provide a link to the Creative Commons license, and indicate if changes were made. The Creative Commons Public Domain Dedication waiver (http://creativecommons.org/publicdomain/zero/1.0/) applies to the data made available in this article, unless otherwise stated. 


\section{Background}

Iliopsoas abscess (IPA) presents an infection with purulent materials appearing within iliopsoas muscle components. It is a rare clinical entity and difficult to diagnose due to its insidious onset and nonspecific symptoms. IPA is classified into primary and secondary types depending on the origin of infectious focus. Primary IPA is originated from an infection of distant source spread through hematological or lymphatic routes. Secondary IPA is an infectious process involving adjacent structures via direct invasion. Staphylococcus aureus is the most common microorganism in primary IPA and secondary IPA related to skeletal muscular infections. Enteric bacteria, such as Escherichia coli, Klebsiella pneumoniae and Salmonella, are the leading microorganisms in secondary IPA caused by intraabdominal infection, and often with mixed polymicrobial presentations [1-4]. Little is known on the association between IPA and CVD. Cardiovascular disorders include primary aortic mycotic aneurysm, endograft infection of the aorta, and infective endocarditis [5-8]. Computed tomography (CT) provides a rather definitive diagnosis of IPA and associated focus of adjacent structure [1-8]. Clinical managements should include empiric antibiotics, percutaneous drainage (PCD), and open surgery for to remove the infectious structures involved [5-8]. When delayed in diagnosis, IPA is a life-threatening condition. The mortality is higher for secondary IPA than for primary IPA, especially those associated with CVD. The mortality reaches up to $100 \%$ if the condition is left untreated [7]. Here, we carried out a hospital-based observational case series study of IPA associated with CVD at a referral medical center in central Taiwan.

\section{Materials and methods}

This study was approved by the institutional review board of Taichung Veterans General Hospital (No. CE18102A). Data were collected from the electronic database of Taichung Veterans General Hospital, a 1520-bed tertiary referral hospital in central Taiwan. Patients were selected for this study based on the ICD-10 codes, including K68.12 (iliopsoas abscess, psoas muscle abscess), I71.4 (abdominal aortic aneurysm), I71.6 (thoracoabdominal aortic aneurysm), I71.9 (aortic aneurysm), I72.9 (mycotic aneurysm), I33.9 (acute endocarditis), and I38 (endocarditis). IPA associated with CVD (primary aortic mycotic aneurysm, stent-graft/endograft infection of the aorta, and infective endocarditis) was confirmed in diagnosis by the classical findings on $\mathrm{CT}$ with contrast media and transesophageal echocardiography (TEE), compatible with clinical presentation, laboratory investigations, and cultures of pus/tissue and blood. Typical CT images of IPA associated with CVD included primary mycotic abdominal aortic aneurysm (AAA) and stent-graft/endograft infection of AAA with abscess within the iliopsoas muscle with or without gas. All CT images were reviewed by the primary study investigator and a radiologist. Infective endocarditis with vegetation was confirmed through TEE by a cardiologist.

The primary outcome of this study was the intrahospital mortality of patients who had been treated for IPA associated with CVD and discharged from hospital with improvement of laboratory data and clinical conditions. During hospitalization, respiratory failure with intubation admitted to ICU with intubation $>3$ days was defined as a clinical complication of IPA associated with CVD. We defined "successful treatment" as the improvement in clinical conditions, in follow-up imaging, and discharged alive after treatment with PCD, surgery, or antibiotics alone. "Failed treatment" was defined as mortality during hospitalization or deterioration of clinical conditions with accompanying non-decremental changes in follow-up imaging, all of which had necessitated another advanced treatment modality (i.e., antibiotics plus PCD, antibiotics plus surgery, or PCD followed by surgery). Patients received a minimum follow-up period of 1 year. Excluded cases were those $\leq 18$ years of age (according to the law in Taiwan, informed consent must be signed by their parents), or had an incomplete treatment course.

Fifteen patients of IPA associated with CVD admitted to our hospital between July 2007 and December 2017 were included in this study. Demographic data, laboratory investigations, etiological pathogens, infectious origins, management approaches, clinical process, and treatment outcome were coded for further statistical analyses. Continuous variables were presented as mean \pm $\mathrm{SD}$, and categorical variables as numbers and percentages. Further comparisons were performed for continuous variables using Mann-Whitney $U$ test and for categorical variables using chi-square test or Fisher's exact test. $P$ values $<0.05$ were considered statistically significant. Analyses were performed using the Statistical Package for the Social Science (IBM SPSS version 22.0; International Business Machines Corp, New York, USA).

\section{Results \\ Clinical features}

Fifteen patients of IPA associated with CVD were enrolled, including 12 males (80\%) and 3 females (20\%), aged $63.2 \pm 16.9$ years (range, $31-85$ years). CVD included stent-graft/endograft infection of AAA (6 patients, 40\%), primary mycotic AAA (5 patients, 33.3\%), and infective endocarditis (4 patients, 26.7\%).

Nine patients $(60 \%)$ had respiratory failure that required intubation and stay in ICU for $>3$ days, and 6 patients $(40 \%)$ had respiratory failure with intubation but the stay in ICU for $\leq 3$ days. Incidence of patients staying 
in the ICU with intubation for over 3 days was 33\% for the survival group and $100 \%(P=0.028)$ for the nonsurvival group. The ICU stay with intubation for $\leq 3$ days $(67 \%$ vs. $0 \%, P=0.028)$ was a good prognostic factor for survival group. The average length of hospital stay was $33.1 \pm 20.5$ days (range, $3-81$ days; median, 33 days). The hospital stay was $42.6 \pm 19.2$ days for the survival group, and $19.0 \pm 14.1$ days $(P=0.018)$ for the non-survival group. The overall intra-hospital mortality rate was $40 \%$. The demographic characteristics were summarized in Table 1.

\section{Laboratory investigations}

The laboratory investigations were summarized in Table 2. Twelve patients (80\%) had systemic inflammatory response syndrome with 2 or more of the following variables: (1) fever of $>38^{\circ} \mathrm{C} \quad\left(100.4{ }^{\circ} \mathrm{F}\right)$ or $<36^{\circ} \mathrm{C}$ $\left(96.8^{\circ} \mathrm{F}\right)$; (2) heart rate of $>90$ beats $/ \mathrm{min}$; (3) respiratory rate of $>20$ breaths/min or arterial $\mathrm{CO}_{2}$ tension $\left(\mathrm{P}_{\mathrm{a}} \mathrm{CO}_{2}\right)$ of < $32 \mathrm{mmHg}$; (4) abnormal white blood cells $(>12,000$ / $\mathrm{mm} 3$ or $<4000 / \mathrm{mm} 3$ or $>10 \%$ immature [band] forms). We found that hypoalbuminemia $(2.9 \pm 0.5$ vs. $2.2 \pm 0.3$, $P=0.009)$ and hyponatremia (132.6 \pm 2.6 vs. $128.8 \pm 3.6$, $P=0.032)$ were poor prognostic factors for the nonsurvival group.

\section{Etiological pathogens}

Results of pus/tissue cultures showed monomicrobial infection in 2 patients, polymicrobial infection in 5 patients, and no growth in 1 patient. No culture of pus/ tissue was available in 7 patients due to treatment with antibiotics only and prior treatment at other rural hospitals. Results of blood culture showed monomicrobial infection in 10 patients (5 with Gram-positive and 5 with Gram-negative), polymicrobial infection in 4 patients, and no growth in 1 patient. The results of culturing pus/ tissue and blood were summarized in Table 3.

\section{Imaging studies}

Of 15 patients, all received CT scan for a definitive diagnosis of IPA associated with CVD and 9 patients underwent TEE. Of 15 patients, depicted on their CT, maximum diameter of objects $>3 \mathrm{~cm}$ was found in 9 patients (60\%), bilateral involvement in 8 patients (53.3\%), and gas-formation in 5 patients (33.3\%). Involving disc/ vertebral body and/or epidural abscess detected by CT was $0 \%$ in the survival group, and $50 \%(P=0.044)$ in the non-survival group. Through TEE in 4 patients, we detected infective endocarditis that involved aortic $(n=2)$ and tricuspid $(n=2)$ valves.

\section{Management and outcomes}

Our approach algorithm of IPA associated with CVD was shown in Fig. 1. On antibiotics-based treatment,
PCD or surgical drainage and combination with endovascular graft or surgical intervention of graft replacement and bypass produced good outcome compared to treatment of antibiotics alone. Conservative treatment with antibiotics was prescribed to patients due to severe infection complicated with unstable hemodynamics or bleeding tendency (thrombocytopenia or prolong PT/ APTT), who were not suitable for PCD or surgical intervention. Aggressive management was carried out for vascular repair and eradiation of infection, including endovascular or surgical repairing of the primary mycotic AAA and stent-graft/endograft infection, and PCD or surgical drainage of IPA according to the clinical conditions. Cumulative survival rate for conservative treatment was $25 \%$ and for aggressive management was 66.3\% $(P=0.038)$ (Fig. 2).

\section{Discussion}

\section{Comparison with the most recent studies}

Here, we found that CVD included infected vascular graft (40\%), primary mycotic AAA (33.3\%), and endocarditis (26.7\%). These results were similar to those by Ouellette et al., on 109 patients of secondary IPA. They reported 15 cases $(13.8 \%)$ of IPA caused by CVD, that included infected vascular graft $(6,40.0 \%)$, infected catheter $(5$, $33.3 \%)$, endocarditis $(3,20.0 \%)$, and infected fistula (1, 6.7\%) [9]. We found the most common microorganism of blood culture beings Gram-positive cocci $(9,60 \%)$, like staphylococci $(6,40 \%)$ and streptococci $(1,6.7 \%)$. Our results were similar to those by Nakamura et al., who reported Gram-positive cocci $(63,50 \%)$ in 126 patients with psoas abscess and pyogenic spondylitis, including staphylococci (51, 40\%) and streptococci (8, 6.3\%) [10]. For IPA caused by CVD, we recommended here treatment with PCD or surgical drainage and combination with graft replacement to eradicate infection foci. Clinical management with PCD and appropriate antibiotic therapy was safe and effective with shorter hospital stay for IPA. Open surgical intervention might be warranted if there was an underlying pathology [9-11]. Risk factors associated with mortality in IPA caused by CVD were found in our present study to include hypoalbuminemia, hyponatremia, involving disc/vertebral body and/or epidural abscess, and ICU stay with intubation $>3$ days. Nakamura et al. also reported that the mortality was related to hypotension, hypoalbuminemia, liver failure, and renal dysfunction in patients with psoas abscess and pyogenic spondylitis [10]. Intra-hospital mortality rate was $40 \%$ in our present report. If patients had cardiovascular disorders, there was a higher risk of mortality in those with psoas abscess or pyogenic spondylitis as reported by Nakamura et al. [10]. We reviewed the following scientific reports regarding the anatomy of iliopsoas muscle, clinical etiology, microbiology, management, and outcome of IPA caused by CVD. 
Table 1 General demographics of 15 patients with iliopsoas abscess associated with cardiovascular disorders

\begin{tabular}{|c|c|c|c|c|c|c|c|}
\hline \multirow{2}{*}{$\overline{\text { Gender }}$} & \multicolumn{4}{|c|}{ Clinical outcome } & \multirow{2}{*}{\multicolumn{2}{|c|}{$\begin{array}{l}\text { Total } \\
(n, \% ; \text { mean } \pm \text { SD) }\end{array}$}} & \multirow[t]{2}{*}{$P$ value } \\
\hline & \multicolumn{2}{|c|}{ Alive $(n, \% ;$ mean \pm SD) } & \multicolumn{2}{|c|}{ Death $(n, \%$; mean \pm SD) } & & & \\
\hline Male & 8 & $(89 \%)$ & 4 & $(67 \%)$ & 12 & $(80 \%)$ & \\
\hline Female & 1 & $(11 \%)$ & 2 & $(33 \%)$ & 3 & $(20 \%)$ & \\
\hline $\mathrm{Age}^{\#}$ & 63.8 & \pm 16.4 & 62.3 & \pm 19.3 & 63.2 & \pm 16.9 & 1.000 \\
\hline Hospital stay (days) & 42.6 & \pm 19.2 & 19.0 & \pm 14.1 & 33.1 & \pm 20.5 & $0.018^{*}$ \\
\hline Complicated condition & & & & & & & $0.028^{*}$ \\
\hline Intubation with intensive care unit (ICU) stay $\leq 3$ days & 6 & $(67 \%)$ & 0 & $(0 \%)$ & 6 & $(40 \%)$ & \\
\hline Intubation with ICU stay $>3$ days & 3 & $(33 \%)$ & 6 & $(100 \%)$ & 9 & $(60 \%)$ & \\
\hline Lactatemia (Lactate $>12 \mathrm{mg} / \mathrm{dl}$ ) & 7 & $(88 \%)$ & 4 & $(80 \%)$ & 11 & $(85 \%)$ & 1.000 \\
\hline Hypoalbuminemia (Albumin < $2.5 \mathrm{~g} / \mathrm{dl}$ ) & 2 & $(22 \%)$ & 6 & $(100 \%)$ & 8 & $(53 \%)$ & $0.007^{* *}$ \\
\hline Anemia (Hemoglobin < $10 \mathrm{~g} / \mathrm{dl}$ ) & 4 & $(44 \%)$ & 3 & $(50 \%)$ & 7 & $(47 \%)$ & 1.000 \\
\hline Leukocytosis (White blood cells > 12,000/(mm³) & 6 & $(67 \%)$ & 4 & $(67 \%)$ & 10 & $(67 \%)$ & 1.000 \\
\hline Left shift phenomenon (Neutrophils > 80\%) & 6 & $(75 \%)$ & 4 & $(67 \%)$ & 10 & $(71 \%)$ & 1.000 \\
\hline Bandemia & 2 & $(22 \%)$ & 2 & $(33 \%)$ & 4 & $(27 \%)$ & 1.000 \\
\hline Thrombocytopenia (Thrombocytes $<140,000 / \mathrm{mm}^{3}$ ) & 2 & $(22 \%)$ & 2 & $(33 \%)$ & 4 & $(27 \%)$ & 1.000 \\
\hline Hyponatremia (Sodium < 135 mEq/l) & 7 & $(78 \%)$ & 5 & $(83 \%)$ & 12 & $(80 \%)$ & 1.000 \\
\hline Hyperkalemia (Potassium > 5.3 mEq/l) & 0 & $(0 \%)$ & 1 & $(17 \%)$ & 1 & $(7 \%)$ & 0.400 \\
\hline Hypokalemia (Potassium < 3.5 mEq/l) & 0 & $(0 \%)$ & 1 & $(17 \%)$ & 1 & $(7 \%)$ & 0.400 \\
\hline \multicolumn{8}{|l|}{ Impaired renal function } \\
\hline Blood urea nitrogen (BUN > $25 \mathrm{mg} / \mathrm{dl}$ ) & 4 & $(44 \%)$ & 2 & $(33 \%)$ & 6 & $(40 \%)$ & 1.000 \\
\hline Creatinine $(\mathrm{Cr}>1.4 \mathrm{mg} / \mathrm{dl})$ & 4 & $(44 \%)$ & 3 & $(50 \%)$ & 7 & $(47 \%)$ & 1.000 \\
\hline BUN/Cr $(>20)$ & 3 & $(33 \%)$ & 3 & $(50 \%)$ & 6 & $(40 \%)$ & 0.622 \\
\hline Impaired liver function (GPT > 50 U/I or GOT > 40 U/I) & 4 & $(44 \%)$ & 1 & $(17 \%)$ & 5 & $(33 \%)$ & 0.580 \\
\hline Hyperglycemia (Blood glucose > 200 mg/dl) & 3 & $(33 \%)$ & 4 & $(67 \%)$ & 7 & $(47 \%)$ & 0.315 \\
\hline Systolic blood pressure $(\mathrm{mmHg})^{\#}$ & 113.7 & \pm 14.8 & 118.2 & \pm 20.9 & 115.5 & \pm 16.9 & 0.768 \\
\hline Diastolic blood pressure $(\mathrm{mmHg})^{\#}$ & 68.4 & \pm 10.7 & 73.2 & \pm 14.0 & 70.3 & \pm 11.9 & 0.479 \\
\hline Heart rate $(\text { beats } / \mathrm{min})^{\#}$ & 105.8 & \pm 19.7 & 103.8 & \pm 17.4 & 105.0 & \pm 18.2 & 0.679 \\
\hline Body temperature $\left({ }^{\circ} \mathrm{C}\right)^{\#}$ & 37.0 & \pm 1.5 & 37.2 & \pm 0.7 & 37.1 & \pm 1.2 & 0.407 \\
\hline Respiratory rate (breaths/min) ${ }^{\#}$ & 18.6 & \pm 1.3 & 19.7 & \pm 2.0 & 19.0 & \pm 1.6 & 0.346 \\
\hline Systemic inflammatory response syndrome & 7 & $(78 \%)$ & 5 & $(83 \%)$ & 12 & $(80 \%)$ & 1.000 \\
\hline Infectious source ${ }^{c}$ & & & & & & & 0.870 \\
\hline Primary mycotic abdominal aortic aneurysm & 4 & $(44 \%)$ & 2 & $(33 \%)$ & 6 & $(40 \%)$ & \\
\hline Stent-graft/endograft infection & 3 & $(33 \%)$ & 2 & $(33 \%)$ & 5 & $(33 \%)$ & \\
\hline Infective endocarditis & 2 & $(22 \%)$ & 2 & $(33 \%)$ & 4 & $(27 \%)$ & \\
\hline Clinical treatment & & & & & & & 0.235 \\
\hline Antibiotics only & 1 & $(11 \%)$ & 3 & $(50 \%)$ & 4 & $(27 \%)$ & \\
\hline Drainage/Surgery & 8 & $(89 \%)$ & 3 & $(50 \%)$ & 11 & $(73 \%)$ & \\
\hline Blood culture ( $n=9$ vs. 5 ) & & & & & & & 0.580 \\
\hline Monomicrobial (Gram-positive and Gram-negative) & 7 & $(78 \%)$ & 3 & $(60 \%)$ & 10 & $(71 \%)$ & \\
\hline Polymicrobial & 2 & $(22 \%)$ & 2 & $(40 \%)$ & 4 & $(29 \%)$ & \\
\hline Pus/Tissue culture ( $n=4$ vs. 3 ) & & & & & & & 0.429 \\
\hline Monomicrobial (Gram-positive only) & 2 & $(50 \%)$ & 0 & $(0 \%)$ & 2 & $(29 \%)$ & \\
\hline Polymicrobial & 2 & $(50 \%)$ & 3 & $(100 \%)$ & 5 & $(71 \%)$ & \\
\hline
\end{tabular}


Table 1 General demographics of 15 patients with iliopsoas abscess associated with cardiovascular disorders (Continued)

\begin{tabular}{|c|c|c|c|c|c|c|c|}
\hline \multirow[b]{3}{*}{ Treatment at other rural hospitals } & \multicolumn{4}{|c|}{ Clinical outcome } & \multirow{2}{*}{\multicolumn{2}{|c|}{$\begin{array}{l}\text { Total } \\
(n, \% ; \text { mean } \pm S D)\end{array}$}} & \multirow{3}{*}{$\begin{array}{c}P \text { value } \\
1.000\end{array}$} \\
\hline & \multicolumn{2}{|c|}{ Alive $(n, \%$; mean \pm SD) } & \multicolumn{2}{|c|}{ Death $(n, \%$; mean \pm SD) } & & & \\
\hline & 2 & $(22 \%)$ & 2 & $(33 \%)$ & 4 & $(27 \%)$ & \\
\hline Maximum diameter of iliopsoas abscess (IPA) & 3.7 & \pm 1.4 & 4.5 & \pm 2.0 & 4.0 & \pm 1.6 & 0.480 \\
\hline Maximum diameter of IPA $>3 \mathrm{~cm}$ & 5 & $(56 \%)$ & 4 & $(67 \%)$ & 9 & $(60 \%)$ & 1.000 \\
\hline Gas-forming & 2 & $(22 \%)$ & 3 & $(50 \%)$ & 5 & $(33 \%)$ & 0.329 \\
\hline Involvement ${ }^{c}$ & & & & & & & 0.683 \\
\hline Right & 3 & $(33 \%)$ & 1 & $(17 \%)$ & 4 & $(27 \%)$ & \\
\hline Left & 2 & $(22 \%)$ & 1 & $(17 \%)$ & 3 & $(20 \%)$ & \\
\hline Bilateral & 4 & $(44 \%)$ & 4 & $(67 \%)$ & 8 & $(53 \%)$ & \\
\hline Multiple lobulated & 9 & $(100 \%)$ & 6 & $(100 \%)$ & 15 & $(100 \%)$ & - \\
\hline Discitis/Paraspinal/Epidural abscess & 0 & $(0 \%)$ & 3 & $(50 \%)$ & 3 & $(20 \%)$ & $0.044^{*}$ \\
\hline Receiving transesophageal echocardiography (TEE) & 6 & $(67 \%)$ & 3 & $(50 \%)$ & 9 & $(60 \%)$ & 0.622 \\
\hline Infective endocarditis found on TEE & 2 & $(22 \%)$ & 2 & $(33 \%)$ & 4 & $(27 \%)$ & 1.000 \\
\hline Recurrence of IPA & 3 & $(33 \%)$ & 0 & $(0 \%)$ & 3 & $(20 \%)$ & 0.229 \\
\hline Tool for follow up & & & & & & & 0.329 \\
\hline Computed tomographic scan & 7 & $(78 \%)$ & 3 & $(50 \%)$ & 10 & $(67 \%)$ & \\
\hline No mentioned or Unknown & 2 & $(22 \%)$ & 3 & $(50 \%)$ & 5 & $(33 \%)$ & \\
\hline Hypertension & 5 & $(56 \%)$ & 3 & $(50 \%)$ & 8 & $(53 \%)$ & 1.000 \\
\hline Diabetes mellitus & 3 & $(33 \%)$ & 4 & $(67 \%)$ & 7 & $(47 \%)$ & 0.315 \\
\hline Cerebrovascular accident & 2 & $(22 \%)$ & 2 & $(33 \%)$ & 4 & $(27 \%)$ & 1.000 \\
\hline Bed-ridden status & 1 & $(11 \%)$ & 3 & $(50 \%)$ & 4 & $(27 \%)$ & 0.235 \\
\hline Malignancy & 2 & $(22 \%)$ & 1 & $(17 \%)$ & 3 & $(20 \%)$ & 1.000 \\
\hline Chronic kidney disease & 3 & $(33 \%)$ & 0 & $(0 \%)$ & 3 & $(20 \%)$ & 0.229 \\
\hline Alcoholism & 2 & $(22 \%)$ & 1 & $(17 \%)$ & 3 & $(20 \%)$ & 1.000 \\
\hline Hemodialysis & 1 & $(11 \%)$ & 0 & $(0 \%)$ & 1 & $(7 \%)$ & 1.000 \\
\hline Intravenous drug abuse & 1 & $(11 \%)$ & 1 & $(17 \%)$ & 2 & $(13 \%)$ & 1.000 \\
\hline
\end{tabular}

${ }^{\#}$ Mann-Whitney $\mathrm{U}$ test. Fisher's exact test. ${ }^{\mathrm{C} C h i-S q u a r e}$ test. ${ }^{*} p<0.05,{ }^{* *} p<0.01$

Continuous data were expressed median and IQR

Categorical data were expressed number and percentage

\section{Anatomy of the iliopsoas muscle}

Psoas muscle is a long fusiform muscle located along the low thoracic, lumbar, and first sacral region of the vertebral column and brim of the lesser pelvis. It joins the iliacus muscle and together forms the iliopsoas muscle (IPM). It lies in close proximity to the sigmoid colon, appendix, jejunum, ureters, abdominal aorta, kidneys, pancreas, spine, and iliac lymph nodes. Hence any infectious process of these organs could extend into the IPM. The abundant blood supply and lymphatic drain of IPM may predispose itself to hematogenous or lymphatic spread from an occult infection at a distant site [1-3].

\section{Definition of iliopsoas abscess}

Iliopsoas abscess (IPA) has been traditionally considered a rare infection with purulent materials developed within the IPM compartment. IPA was first described by Mynter in 1881 [1, 2, 4]. Pyogenic IPA is divided into two types: primary and secondary. Primary IPA develops most likely secondary to Staphylococcal bacteremia from an occult infection in the body owing to the rich vascular supply and lymphatic drain of IPM. It is predominantly seen in younger patients and in the developing and tropical countries (Asia and Africa). Secondary IPA is caused by contiguous spread from an infectious process of the adjacent structures (gastrointestinal tract, urinary tract, vessel, and spine). The condition is commonly found in Europe and North America, with the mixed enteric bacteria being the major microorganisms involved [1, 2, 5-13]. Primary and secondary IPA occurred in a frequency ratio of 11 to $89 \%$, respectively [13].

\section{Mechanisms and risk factors of iliopsoas abscess and cardiovascular disorders}

Spondylodiscitis associated with the epidural, paraspinal, and psoas abscesses are caused by hematogenous or 
Table 2 Laboratory investigations of 15 patients with iliopsoas abscess associated with cardiovascular disorders

\begin{tabular}{|c|c|c|c|c|c|c|c|}
\hline \multirow[b]{3}{*}{ White blood cells $\left(\times 10^{3} / \mathrm{mm}^{3}\right)^{\#}$} & \multicolumn{4}{|c|}{ Clinical outcome } & \multirow{2}{*}{\multicolumn{2}{|c|}{$\begin{array}{l}\text { Total } \\
(n=15 ; \text { mean } \pm S D)\end{array}$}} & \multirow{3}{*}{$\begin{array}{l}P \text { value } \\
0.906\end{array}$} \\
\hline & \multicolumn{2}{|c|}{ Alive $(n=9 ;$ mean \pm SD $)$} & \multicolumn{2}{|c|}{ Death $(n=6$; mean \pm SD) } & & & \\
\hline & 14.1 & \pm 5.5 & 16.8 & \pm 9.1 & 15.2 & \pm 7.0 & \\
\hline Neutrophils (\%) ${ }^{\#}$ & 84.3 & \pm 10.5 & 81.9 & \pm 13.5 & 83.3 & \pm 11.4 & 0.860 \\
\hline Band $(\%)^{\#}$ & 2.6 & \pm 5.1 & 0.8 & \pm 1.6 & 1.9 & \pm 4.1 & 0.880 \\
\hline Hemoglobin $(\mathrm{g} / \mathrm{dl})^{\#}$ & 10.9 & \pm 2.2 & 11.1 & \pm 3.1 & 11.0 & \pm 2.5 & 0.860 \\
\hline Thrombocytes $\left(\times 10^{3} / \mathrm{mm}^{3}\right)^{\#}$ & 241.4 & \pm 191.8 & 188.0 & \pm 114.5 & 220.1 & \pm 162.6 & 0.480 \\
\hline Albumin $(\mathrm{g} / \mathrm{dl})^{\#}$ & 2.9 & \pm 0.5 & 2.2 & \pm 0.3 & 2.6 & \pm 0.6 & $0.009^{* *}$ \\
\hline Alkaline phosphatase $(U / I)^{\#}$ & 189.0 & \pm 83.3 & 281.7 & \pm 195.2 & 226.1 & \pm 140.7 & 0.316 \\
\hline Glutamic oxaloacetic transaminase $\left(\mathrm{U} / \mathrm{I}^{\#}\right.$ & 38.7 & \pm 27.8 & 32.8 & \pm 14.5 & 36.6 & \pm 23.4 & 0.640 \\
\hline Glutamic pyruvic transaminase $(\mathrm{U} /)^{\#}$ & 45.2 & \pm 33.4 & 38.5 & \pm 18.7 & 42.5 & \pm 27.8 & 0.953 \\
\hline Lactic dehydrogenase $\left(\mathrm{U} / \mathrm{I}^{\#}\right.$ & 260.4 & \pm 87.0 & 246.2 & \pm 117.3 & 255.4 & \pm 94.6 & 0.739 \\
\hline C-reactive protein $(\mathrm{mg} / \mathrm{dll})^{\#}$ & 19.2 & \pm 9.7 & 15.5 & \pm 5.4 & 17.7 & \pm 8.2 & 0.409 \\
\hline Glucose $(\mathrm{mg} / \mathrm{dll})^{\#}$ & 192.0 & \pm 85.6 & 223.0 & \pm 126.5 & 204.4 & \pm 100.8 & 1.000 \\
\hline Lactate $(\mathrm{mg} / \mathrm{dl})^{\#}$ & 26.2 & \pm 25.4 & 38.4 & \pm 45.4 & 30.9 & \pm 33.2 & 0.558 \\
\hline Sodium $(\mathrm{mEq} / \mathrm{l})^{\#}$ & 132.6 & \pm 2.6 & 128.8 & \pm 3.6 & 131.1 & \pm 3.5 & $0.032^{*}$ \\
\hline Potassium $\left(\mathrm{mEq} / \mathrm{I}^{\#}\right.$ & 4.0 & \pm 0.4 & 4.7 & \pm 0.9 & 4.3 & \pm 0.7 & 0.058 \\
\hline Blood urea nitrogen $(\mathrm{mg} / \mathrm{dl})^{\#}$ & 44.0 & \pm 59.2 & 23.8 & \pm 9.2 & 35.9 & \pm 46.2 & 0.953 \\
\hline Creatinine $(\mathrm{mg} / \mathrm{dll})^{\#}$ & 2.1 & \pm 2.3 & 1.4 & \pm 0.4 & 1.8 & \pm 1.8 & 0.723 \\
\hline $\mathrm{pH}^{\#}$ & 7.4 & \pm 0.1 & 7.5 & \pm 0.1 & 7.4 & \pm 0.1 & 0.637 \\
\hline $\mathrm{P}_{\mathrm{a}} \mathrm{CO}_{2}(\mathrm{mmHg})^{\#}$ & 35.1 & \pm 9.7 & 32.8 & \pm 8.5 & 34.2 & \pm 9.0 & 0.637 \\
\hline $\mathrm{HCO}_{3}{ }^{-}(\mathrm{mEq} / \mathrm{l})^{\#}$ & 22.4 & \pm 6.1 & 22.8 & \pm 7.0 & 22.6 & \pm 6.2 & 0.814 \\
\hline
\end{tabular}

${ }^{\#}$ Mann-Whitney U test. Fisher's exact test. ${ }^{c}$ Chi-Square test. ${ }^{*} p<0.05,{ }^{* *} p<0.01$

Continuous data were expressed median and IQR

Categorical data were expressed number and percentage

Table 3 Microorganisms isolated from 15 patients of iliopsoas abscess associated with cardiovascular disorders

\begin{tabular}{|c|c|c|}
\hline Microorganisms & Culture of pus/tissue $(n, \%)$ & Culture of blood $(n, \%)$ \\
\hline Gram-positive & $5(33.3 \%)$ & $9(60 \%)$ \\
\hline Staphylococcus aureus & $3(20 \%)$ & $6(40 \%)$ \\
\hline Streptococcus mitis group & $1(6.7 \%)$ & $1(6.7 \%)$ \\
\hline Cutibacterium acnes (Propionibacterium acnes)^ & $1(6.7 \%)$ & $0(0 \%)$ \\
\hline Bacillus cereus & $0(0 \%)$ & $1(6.7 \%)$ \\
\hline Listeria monocytogenes $\%$ & $0(0 \%)$ & $1(6.7 \%)$ \\
\hline Gram-negative & $5(33.3 \%)$ & $8(53.3 \%)$ \\
\hline Salmonella & $1(6.7 \%)$ & $4(26.7 \%)$ \\
\hline Acinetobacter baumannii & $1(6.7 \%)$ & $0(0 \%)$ \\
\hline Escherichia coli & $1(6.7 \%)$ & $1(6.7 \%)$ \\
\hline Klebsiella pneumoniae & $1(6.7 \%)$ & $1(6.7 \%)$ \\
\hline Prevotella sp.^* & $1(6.7 \%)$ & $0(0 \%)$ \\
\hline Pseudomonas aeruginosa & $0(0 \%)$ & $1(6.7 \%)$ \\
\hline Veillonella sp.^^ & $0(0 \%)$ & $1(6.7 \%)$ \\
\hline Fungus & $2(13.3 \%)$ & $1(6.7 \%)$ \\
\hline Candida ${ }^{\#}$ & $2(13.3 \%)$ & $1(6.7 \%)$ \\
\hline
\end{tabular}

1. $\wedge$ presented anaerobic; ${ }^{\%}$ presented facultative anaerobic

$2 .{ }^{\$}$ including Salmonella enterica serovar Typhimurium and Salmonella enterica serovar Enteritidis"; * including $P$. melaninogenica and $P$. oralis; ${ }^{*}$ including $C$. tropicalis, C. albicans, and unclassified Candida sp.

3. Polymicrobial infections of pus/tissue and blood were found in 5 and 4 patients, respectively 


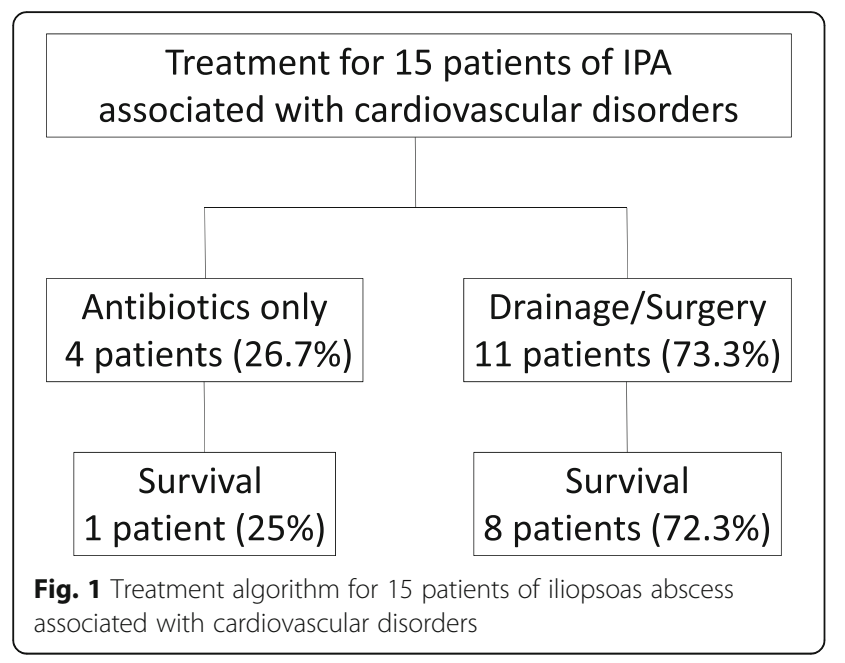

lymphatic spread, and a direct extension from the adjacent focus like infective endocarditis and infected aortic aneurysm or endograft [14-18]. Patients with IPA should be investigated for infective endocarditis, particularly caused by Staphylococcus aureus [16]. Emphysematous IPA is an unusual complication of mycotic AAA. Progressive inflammatory process extending beyond the arterial wall with a point of leakage in the arterial adventitia into the surrounding IPM may result in a localized abscess with gas collection and may penetrate the arterial adventitia leading to retroperitoneal hemorrhage and sudden death [18-20]. The possible mechanisms of mycotic aneurysm include bacteremia with septic emboli, which enter the vasa vasorum and cause vessel-wall infection leading to aneurysm formation, systemic pathogens inoculating in the existing aortic aneurysm, and aneurysm formation from an extravascular source or adjacent tissues $[19,21,22]$.

The predisposing factors for the development of aortic infective aneurysms are old age ( $\geq 65$ years), pre-existing aneurysm, atherosclerotic disease, diabetes mellitus (DM), hypertension, hyperlipidemia, rheumatoid arthritis, malignancy, and acquired immune deficiency syndrome. The intimal damage and atherosclerosis promote the development of mycotic aneurysm [18, 21]. DM $(64 \%)$ is the dominant predisposing or associated factor of IPA. Autonomic neuropathy of small bowel reduces intestinal motility in diabetic patients, raises intestinal transit time and therefore increases the risk of Salmonellosis associated with IPA and infected aortic aneurysm $[7,23]$. In our present study, the incidence of old age ( $\geq 65$ years) was $80 \%$, hypertension $53.3 \%$, DM $46.7 \%$, and Salmonellosis $26.7 \%$.

\section{Incidence and the association between iliopsoas abscess and cardiovascular disorders}

IPA is a rare condition with an incidence of $0.4 / 100,000$ in the UK [5]. Infection rates of IPA have increased from 0.5 cases/10,000 admissions to 6.5 cases/10,000 admissions [2].

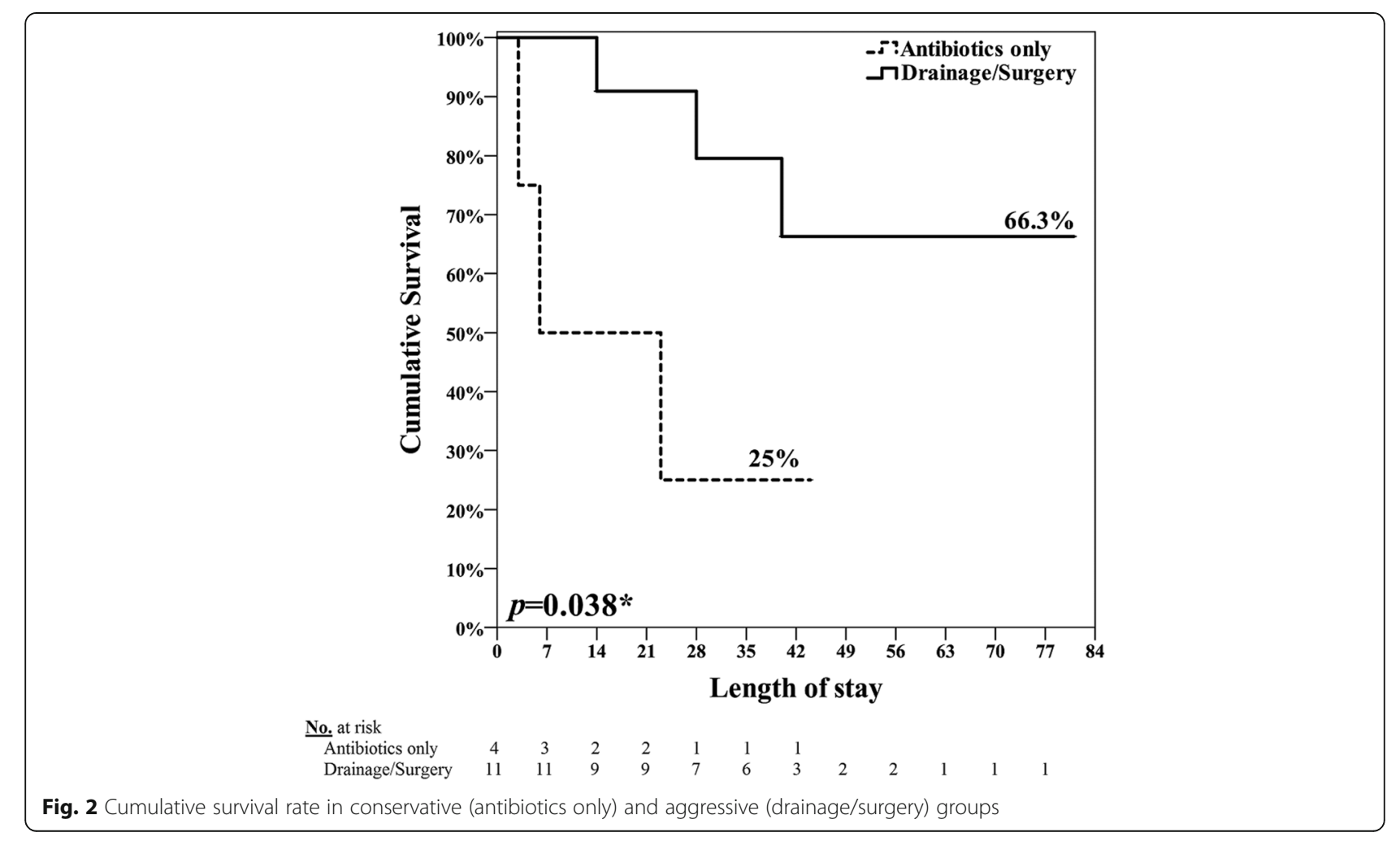


However, IPA likely has a rate $>12$ cases/year [24]. More than a thousand cases have been reported in review the literature $[25,26]$. However, cases of IPA are likely underestimated as some IPAs are underdiagnosed or unreported before mortality or appeared in nonEnglish literature.

Mycotic aortic aneurysm is a rare condition with 1$3 \%$ incidence from all aortic aneurysms [19, 27]. Stentgraft/endograft infection of the aorta is a rare clinical entity with an incidence of $0.5-3 \%$ in the conventional open surgery repair and $0.1 \%(6.2 / 1000$ person years) in the endovascular repair $[15,28-31]$. The incidence of aortic mycotic aneurysm resulting in IPA is $1.4 \%$ [20, 30]. In 606 patients with infective endocarditis, $4.6 \%$ has pyogenic vertebral osteomyelitis [14].

IPA associated with vascular origins, including infective endocarditis, primary aortic mycotic aneurysm, and endograft infection of aorta, has been rarely reported at an incidence of 5\% [2]. The association between IPA and primary aortic mycotic aneurysm is extremely rare with a frequency of $4 \%[6,20,31,32]$. The incidence of endograft infection after endovascular repair of AAA is 0.69\% in an analysis of 1302 patients [33]. IPA secondary to graft infection after aortoiliac surgery or infection of endovascular positioned stents is an uncommon clinical presentation. The incidence of IPA caused by prosthetic stent graft infection after endovascular repair of AAA is $0.39 \%$ in a study of 509 patients [29]. Acute pyogenic IPA is predominant in females [7]. Primary mycotic AAA in 6 patients (40\%), stent-graft/endograft infection in 5 patients (33.3\%), and infective endocarditis in 4 patients $(26.7 \%)$ have been reported. IPA associated with CVD is more common in males, and observation that is consistent with a $4: 1$ ratio of male-to-female as found in our present study. The possible reason could be related to more cardiovascular disorders in males.

\section{Clinical presentation of iliopsoas abscess associated with cardiovascular disorders}

Common clinical features include flank/back pain, vague abdominal pain, fever, limp, malaise, weight loss, and groin lump [5]. In a study of 61 IPA patients, features are observed with pain (95\%), gastrointestinal tract complaints (43\%), and lower extremity pain (30\%) [13]. Three quarter $(76 \%)$ of patients with IPA have pain in the abdomen, flank or back [7]. The classical clinical triad of fever, back/flank pain, and limited hip movement is present in only $30 \%$ of patients $[1,5,8]$. Half of patients with Salmonellosis of abdominal aorta have the clinical triad of fever, pulsatile abdominal mass, and back pain [23]. Clinical presentation of infected endograft of aorta includes positive blood and/or tissue culture, perigraft fluid with presence of air, and associated IPA or groin abscess on image study [15, 27, 34].

\section{Diagnosis of iliopsoas abscess associated with cardiovascular disorders}

IPA is a relatively uncommon condition that can present itself with vague clinical presentation. Its insidious onset and occult features can delay diagnosis, resulting in high mortality and morbidity [1]. Clinical investigations of IPA should include radiology and cultures of blood, pus/ tissue, and urine. IPA is commonly diagnosed via modern imaging, such as ultrasonography, CT, and magnetic resonance imaging (MRI). CT is the "gold standard" for a definitive diagnosis of IPA, although MRI can provide better discrimination of soft tissues, allowing visualization of the abscess wall and the surrounding structures. CT scan is the most sensitive and can confirm the diagnosis and define the extension of IPA [1, 3, 7, 13]. The features of IPA on CT include an enlarged IPM, with a rounded contour with contrast enhanced rim of the abscess wall, a relatively low-density area, and gas within IPM [6].

The diagnosis of mycotic aneurysm is usually depending on the clinical presentation of fever, abdominal or chest pain, positive blood cultures, and a pulsatile mass [19]. CT angiography (CTA) of the aorta or Multidetector CT (MDCT) is the modality of choice for the vascular evaluation to detect the mycotic aneurysms and the infected endograft as an early stage. Clinical characteristics of infected aortic aneurysm or stent-graft/endograft include eccentric contour, saccular shape (especially lobulated), rapid expansion or development with periaortic/perigraft soft tissue stranding, free fluid, air pockets, and abnormal adjacent structures (vertebral body destruction, discitis, and IPA) $[6,20,23,27,35,36]$. CT is the most reliable diagnostic mandatory tool for IPA related to CVD with a perfect sensitivity of $100 \%$. Bilateral IPA in $13 \%$ patients and multiple IPAs in 25\% patients are diagnosed based on CT [13]. Periodic CT scans (yearly) are recommended after endovascular repair and therefore may enable more rapid detection of gas or fluid, suggesting infection [33]. Abdominal CT depicted air within the endograft and emphysematous abscess of the right IPM in our index case of 75-year-old man with a history of an endograft implantation for AAA with an involvement of bilateral iliac arteries (Fig. 3).

Laboratory investigations are non-specific, but high levels of white blood cell count and C-reactive protein, anemia, and high erythrocyte sedimentation rates provide clinical clues of associated infection $[1,5,20,22]$.

\section{Microbiology of iliopsoas abscess associated with cardiovascular disorders}

The most common organism in IPA with skeletal infection, regardless of primary IPA and secondary, is Staphylococcus aureus. In secondary IPA, most origins are from the gastrointestinal and urinary tracts, with Escherichia coli being the most common organism. 


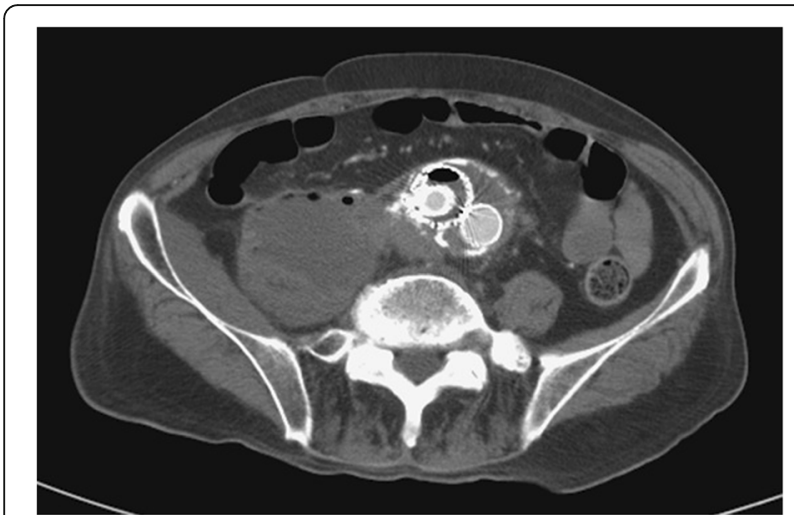

Fig. 3 Abdominal CT depicted air within the endograft and emphysematous abscess of the right IPM

Other organisms have also been case-reported, involving Bacteroides species, Mycobacterium tuberculosis, Enterococcus faecalis, Peptostreptococcus, Viridans group streptococci, and methicillin resistant Staphylococcus aureus [5]. In Taiwan, urinary tract infection (52\%) of enteric microorganisms (44\% of Escherichia coli and 24\% of Klebsiella spp.) is the most common infection source of secondary IPA and typically found in older, female, and diabetic patients. Klebsiella pneumoniae has been proposed as an independent factor for IPA mortality in Taiwan $[7,8,26]$.

A positive culture rate of $64.7-75 \%$ is a definitive microbial diagnosis of IPA $[2,5,12]$. Combination of blood and abscess cultures can confirm $>67 \%$ of patients with a definitive pathogen diagnosis of IPA [2]. However, blood culture can be negative for IPA in as many as $47 \%$ of patients [20]. Prior antibiotic use (inpatient antimicrobial therapy and antibiotics prior to their drainage procedure in emergency department) may have lowered the yield of culture data or may have sterilized the infection site [2].

About 5\% of cultures from aortic thrombus obtained during open aneurysm repair has positive findings of Gram-positive and negative bacteria [37]. Most reported cases of IPA related to aortic mycotic aneurysm are caused by Salmonella infection [3]. Endovascular infection is a serious complication of non-typhoid Salmonella which is specifically linked to atheroscerlosis in Taiwan, particularly infrarenal abdominal aortic infected aneurysms and endocarditis [8, 38]. Salmonella is responsible for $27-75 \%$ of infected aortic aneurysm. Salmonella enterica serovar Typhimurium and Salmonella enterica serovar Choleraesuis are the species most commonly isolated. Other causative organisms are mycobacterium, Gram-negative bacilli other than Salmonella, mixed bacteria, and fungi [21, 23, 33, 39].

In aortic graft infections, the most common organisms are Gram-positive cocci (Staphylococcus and Streptococcus species are the most prevalent), although Gram- negative organisms, such as Escherichia coli and Bacteroides species, have also been isolated [40]. Only $68 \%$ of cases show a positive result in culture for a definitive microbial diagnosis of aortic graft infection. The most common culture reported in the literature is Grampositive bacteria, with the specific bacterial organism Staphylococcus spp. representing 23-88\% of the entire positive cultures [33]. In patients of infected aortic stent-graft/endograft, $42.1 \%$ have monomicrobial infections, and $57.9 \%$ polymicrobial infections. Staphylococcus (63.2\%) and Streptococcus (31.6\%) are the most common bacteria [34].

\section{Treatment of iliopsoas abscess associated with cardiovascular disorders}

Treatment of IPA consists of application of appropriate antibiotics along with abscess drainage. For primary IPA, antistaphylococcal antibiotics should be started even before knowing the culture results. For secondary IPA, broad-spectrum antibiotics against mixed enteric bacteria should be used. Antibiotics should be properly adjusted according to the results of the abscess fluid culture and drug sensitivity. PCD through sonography/ CT-guide or surgical drainage is suggested to treat IPA, especially for the primary type. PCD is less invasive and is considered the first choice. Open surgery is recommended in the case of the following: PCD failure, relative contraindication of $\mathrm{PCD}$, and the presence of intraabdominal pathology which requires surgery $[1,41]$. Consideration maybe given to pathogen-directed antimicrobial coverage for Gram-positive cocci in cases of primary IPA and in secondary IPA of skeletal origins, and polymicrobial coverage in cases of IPAs spreading from urinary tract or of gastrointestinal origin [2].

In the case of second stage with open definitive surgery to treat an infected AAA, endovascular stent graft should be considered as a bridge with an implantation of the endoprosthesis below the level of the bilateral renal arteries to prevent persistent aneurysmal infection [22, 27, 42]. The advantages of endovascular repair for infected AAA have reduced risks of bacterial spread and graft infections, speeding up recovery, and lowering care costs, especially for those critically sick. The cumulative rate of late conversion to open repair is $2 \%$ /year and the risk of aneurysm rupture is $1 \%$ /year $[22,31]$.

A combination of early PCD, debridement and resection of the surrounding tissue, aortic reconstruction with graft replacement or extra-anatomic bypass, and targeted long-term antibiotic treatment is mandatory protocol for IPA associated with CVD. The recommended duration of antibiotics use is $4-6$ weeks, and in accordance with the clinical conditions [2, 8, 18, 21, 29, 32-34].

Prognosis and Mortality of iliopsoas abscess associated with cardiovascular disorders. 
The mortality rate of IPA associated with CVD is $40 \%$ in our present study. Gas-formation is an important factor of clinical outcome for patients of IPA. Their mortality rate is $44.0 \%$ for the emphysematous IPA and $16.4 \%$ for the non-emphysematous IPA [41]. If Klebsiella pneumoniae is the microorganism of IPA, the mortality rate is $26-44 \%$. The mortality rate is up to $100 \%$ if IPA is left untreated $[7,26,41]$.

Patients of secondary IPA have longer hospital stay [3]. Patients of IPA associated with an infected aortic aneurysm have a higher incidence of emergency operation, hospital mortality, prosthetic graft infection, and aneurysm-related mortality [8]. Emphysematous IPA may be a clinical clue for ruptured mycotic aneurysm [19]. The aneurysm-related mortality rate is $57 \%$ in patients of IPA associated with aortic mycotic aneurysm. The condition is reportedly one major risk factor for patients with aortic mycotic aneurysm [39].

\section{Limitations of our present study on iliopsoas abscess associated with cardiovascular disorders}

The major limitation of our study is its retrospective nature, small sample size and the selection of management. The infrequent nature of IPA limits our freedom to perform a prospective randomized control study. However, ours is the largest series study so far on IPA associated with CVD.

\section{Conclusion}

Due to tis high mortality rate, clinicians should keep a high suspicion index for IPA associated with CVD through clinical presentation and physical examination. Clinicians should make a correct diagnosis via CT, CTA or MDCT to demonstrate IPA and associated infected vessels or endograft infection. Aggressive management with intervention produced outcomes better than conservative treatment with antibiotics only. Timely empiric antibiotics for common bacteria, PCD or surgical drainage for IPA, endovascular repair, or vascular reconstruction by graft replacement or bypass with intensive care are recommended mandatory to shorten hospital stay, reduce medical costs, and lower mortality rate.

\section{Abbreviations \\ AAA: Abdominal aortic aneurysm; CT: Computed tomographic; CTA: Computed tomographic angiography; CVD: Cardiovascular disorders; DM: Diabetes mellitus; ICU: Intensive care unit; IPA: lliopsoas abscess; IPM: lliopsoas muscle; MDCT: Multi-detector computed tomographic; MRI: Magnetic resonance imaging; PCD: Percutaneous drainage; TEE: Transesophageal echocardiography}

\section{Acknowledgements}

We would like to thank the Clinical Informatics Research \& Development Center and Biostatistics Task Force of Taichung Veterans General Hospital.

\section{Authors' contributions}

SYH, YTC, and MSH conceived and designed the study, analyzed the data, wrote the paper, prepared figures and/or tables. CLT and CAT performed the clinical surgery and intensive care. $\mathrm{CCH}, \mathrm{CYH}$ and $\mathrm{CHS}$ contributed collection of materials and data analysis. YZC conceived and designed the study and reviewed drafts of the paper. M-S Hsieh is an equal contributor to the first author. Y-Z Chang is an equal contributor to the correspondence author. All authors have read and approved the manuscript.

\section{Funding}

We received no specific grant from any funding agency in the public, commercial, or not-for-profit sectors.

\section{Availability of data and materials}

Readers could access the data and material supporting the conclusions of the study by contacting Sung-Yuan Hu at song9168@pie.com.tw.

Ethics approval and consent to participate

All procedures performed were in accordance with the ethical standards of the institutional and/or national research committee and with the 1964 Helsinki declaration and its later amendments or comparable ethical standards. This study was approved by the Institutional Review Board of Taichung Veterans General Hospital (No. CE18102A). We confirmed that informed consent obtained to participate in this study was written or verbal from participants (or their parent or guardian).

\section{Consent for publication}

We confirmed that participants (or their parent or guardian) gave informed consent for publication through written or verbal consent.

\section{Competing interests}

The authors declare that they have no competing interests.

\section{Author details}

${ }^{1}$ Institute of Medicine, Chung Shan Medical University, No. 110, Sec. 1, Jianguo N. Rd, Taichung City 40201, Taiwan. ${ }^{2}$ School of Medicine, Chung Shan Medical University, Taichung City, Taiwan. ${ }^{3}$ Department of Emergency Medicine, Taichung Veterans General Hospital, 1650 Taiwan Boulevard Sect. 4, Taichung City 40705, Taiwan. ${ }^{4}$ Department of Nursing, College of Health, National Taichung University of Technology and Science, Taichung City, Taiwan. ${ }^{5}$ Department of Nursing, Central Taichung University of Technology and Science, Taichung City, Taiwan. ${ }^{6}$ School of Medicine, National Yang-Min University, Taipei City, Taiwan. ${ }^{7}$ Department of Nursing, Jen-Teh Junior College of Medicine, Nursing and Management, Miao-Li County, Taiwan. ${ }^{8}$ Department of Emergency Medicine, Taipei Veterans General Hospital, Tao-Yuan Branch, No. 100, Sec. 3, Cheng-Kung Road, Taoyuan 330, Taiwan. ${ }^{9}$ Department of Emergency Medicine, Taipei Veterans General Hospital, Taipei City, Taiwan. ${ }^{10}$ Institute of Occupational Medicine and Industrial Hygiene, College of Public Health, National Taiwan University, Taipei City, Taiwan. ${ }^{11}$ Department of Medical Research, Taichung Veterans General Hospital, Taichung City, Taiwan. ${ }^{12}$ Division of Infectious Disease, Department of Internal Medicine, Taichung Veterans General Hospital, Taichung City, Taiwan. ${ }^{13}$ Division of Cardiovascular Surgery, Department of Surgery, Taichung Veterans General Hospital, Taichung City, Taiwan. ${ }^{14}$ Department of Clinical Laboratory, Drug Testing Center, Chung-Shan Medical University Hospital, Taichung, Taiwan.

Received: 8 September 2018 Accepted: 29 August 2019 Published online: 25 October 2019

\section{References}

1. Mallick IH, Thoufeeg MH, Rajendran TP. lliopsoas abscesses. Postgrad Med J. 2004;80:459-62.

2. Alonso CD, Barclay S, Tao X, Auwaerter PG. Increasing incidence of iliopsoas abscesses with MRSA as a predominant pathogen. J Inf Secur. 2011;63:1-7.

3. Wong OF, Ho PL, Lam SK. Retrospective review of clinical presentations, microbiology, and outcomes of patients with psoas abscess. Hong Kong Med J. 2013;19:416-23.

4. Mynter H. Acute psoitis. Buffalo Med Surg J. 1881;21:202-10.

5. Shields D, Robinson P, Crowley TP. Iliopsoas abscess - a review and update on the literature. Int J Surg. 2012;10:466-9.

6. Inufusa A, Mikawa Y, Morita I, Fujiwara T. Ruptured abdominal aortic aneurysm associated with a psoas abscess. Arch Orthop Trauma Surg. 2002; 122:306-7. 
7. Huang JJ, Ruaan MK, Lan RR, Wang MC. Acute pyogenic iliopsoas abscess in Taiwan: clinical features, diagnosis, treatments and outcome. J Inf Secur. 2000;40:248-55.

8. Hsu RB, Lin FY. Psoas abscess in patients with an infected aortic aneurysm. J Vasc Surg. 2007;46:230-5.

9. Ouellette L, Hamati M, Flannigan M, Singh M, Bush C, Jones J. Epidemiology of and risk factors for iliopsoas abscess in a large community-based study. Am J Emerg Med. 2019;37:158-9.

10. Nakamura T, Morimoto T, Katsube K, Yamamori Y, Mashino J, Kikuchi K. Clinical characteristics of pyogenic spondylitis and psoas abscess at a tertiary care hospital: a retrospective cohort study. J Orthop Surg Res. 2018;13:302.

11. Benkhadoura MO, El-Mogassabi AH, Mansor SM, et al. lliopsoas abscess: clinical presentation, management, and outcome. Int Surg J. 2019;6:17-21.

12. Kim YJ, Yoon JH, Kim SI, Wie SH, Kim YR. Etiology and outcome of iliopsoas muscle abscess in Korea; changes over a decade. Int J Surg. 2013:11:1056-9.

13. Tabrizian P, Nguyen SQ, Greenstein A, Rajhbeharrysingh U, Divino CM. Management and treatment of iliopsoas abscess. Arch Surg. 2009;144:946-9.

14. Kim JH, Kim SK, Kim DC, et al. Recurrent infective endocarditis associated with pyogenic spondylodiskitis. Korean Circ J. 2011:41:167-70.

15. de Koning HD, van Sterkenburg SM, Pierie ME, Reijnen MM. Endovascular abdominal aortic aneurysm repair complicated by spondylodiscitis and iliaco-enteral fistula. J Vasc Surg. 2008:47:1330-2.

16. Tsuboi I, Yumoto T, Toyokawa T, et al. Staphylococcus aureus bacteremia complicated by ppsoas abscess and infective endocarditis in a patient with atopic dermatitis. Case Rep Infect Dis. 2017;2017:4920182.

17. Farooq Z, Devenney-Cakir B. Clinical case report: discitis osteomyelitis complicated by inferior vena cava venous thrombosis and septic pulmonary emboli. Radiol Case Rep. 2016;11:370-4

18. Carnevalini M, Faccenna F, Gabrielli R, et al. Abdominal aortic mycotic aneurysm, psoas abscess, and aorto-bisiliac graft infection due to Salmonella typhimurium. J Infect Chemother. 2005;11:297-9.

19. Chen CW, Ko WC, Sung JM, Huang JJ. Ruptured mycotic aneurysm of the iliac artery complicated by emphysematous psoas muscle abscess: report of two cases. J Formos Med Assoc. 2002;101:144-7.

20. Macedo TA, Stanson AW, Oderich GS, Johnson CM, Panneton JM, Tie ML. Infected aortic aneurysms: imaging findings. Radiology. 2004;231:250-7.

21. Chen IM, Chang HH, Hsu CP, Lai ST, Shih CC. Ten-year experience with surgical repair of mycotic aortic aneurysms. J Chin Med Assoc. 2005:68:265-71.

22. Koeppel TA, Gahlen J, Diehl S, Prosst RL, Dueber C. Mycotic aneurysm of the abdominal aorta with retroperitoneal abscess: successful endovascular repair. J Vasc Surg. 2004;40:164-6.

23. Learch TJ, Sakamoto B, Ling AC, Donovan SM. Salmonella spondylodiscitis associated with a mycotic abdominal aortic aneurysm and paravertebral abscess. Emerg Radiol. 2009;16:147-50.

24. Gruenwald I, Abrahamson J, Cohen O. Psoas abscess: case report and review of the literature. J Urol. 1992;147:1624-6.

25. Thomas A, Albert AS, Bhat S, Sunil KR. Primary psoas abscess--diagnostic and therapeutic considerations. Br J Urol. 1996;78:358-60.

26. Lai YC, Lin PC, Wang WS, Lai J. An update on psoas muscle abscess: an 8year experience and review of literature. Int J Gerontol. 2011;5:75-9.

27. Gülcü A, Gezer NS, Uğurlu ŞB, Göktay AY. An aortoenteric fistula arising after endovascular management of a mycotic abdominal aortic aneurysm complicated with a psoas abscess. Iran J Med Sci. 2016:41:350-3.

28. Jackson MR, Clagett GP. Aortic graft infection. In: Cronenwett JL, Rutherford RB, editors. Decision making in vascular surgery Philadelphia: W.B. Saunders; 2001. p. 186-91.

29. Sharif MA, Lee $B$, Lau LL, et al. Prosthetic stent graft infection after endovascular abdominal aortic aneurysm repair. J Vasc Surg. 2007;46:442-8.

30. Moussa O, Sreedharan L, Poels J, Ojimba T. Psoas abscess complicating endovascular aortic aneurysm repair. J Surg Case Rep. 2012;2012:16.

31. Dattilo JB, Brewster DC, Fan CM, et al. Clinical failures of endovascular abdominal aortic aneurysm repair: incidence, causes, and management. J Vasc Surg. 2002:35:1137-44.

32. Melissano G, Civilini E, Papa M, Del Guercio R, Chiesa R. Antalgic flexion of the lower limb: an unusual presentation of aortoiliac infection with psoas muscle abscess. Vasc Endovasc Surg. 2005;39:287-92

33. Laser A, Baker N, Rectenwald J, Eliason JL, Criado-Pallares E, Upchurch GR Jr. Graft infection after endovascular abdominal aortic aneurysm repair. J Vasc Surg. 2011;54:58-63.

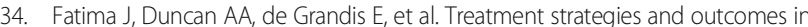
patients with infected aortic endograft. J Vasc Surg. 2013;58:371-9.
35. Abu Bakar A, Ngiu CS, Mohamad Said MS, Periyasamy P. Salmonella related mycotic aneurysm with psoas and Paraortic abscess treated conservatively. Ann Acad Med Singap. 2011;40:467-8.

36. Ahmed MZ, Ling L, Ettles DF. Common and uncommon CT findings in rupture and impending rupture of abdominal aortic aneurysms. Clin Radiol. 2013;68:962-71.

37. Lawrence PF. Conservative treatment of aortic graft infection. Semin Vasc Surg. 2011;24:199-204

38. Cheng WL, Li CW, Li MC, Lee NY, Lee CC, Ko WC. Salmonella infective endocarditis. J Microbiol Immunol Infect. 2016;49:313-20

39. Lin $\mathrm{CH}$, Hsu RB. Primary infected aortic aneurysm: clinical presentation, pathogen, and outcome. Acta Cardiol Sin. 2014;30:514-21.

40. Shalan A, Wilson N, Poels J, Ikponmwosa A, Cavanagh S. The case of the neighbour's cat causing a symptomatic (mycotic) aortic aneurysm and an infected endograft. EJVES Short Rep. 2017;37:18-21.

41. Hsieh MS, Huang SC, Loh e-W, et al. Features and treatment modality of iliopsoas abscess and its outcome: a 6-year hospital-based study. BMC Infect Dis. 2013;13:578

42. Kondo N, Tamura K, Sakaguchi T, Chikazawa G, Yoshitaka H. Replacement of the thoracoabdominal aorta after endovascular abdominal aneurysm repair for ruptured infected aneurysm: a case report. Ann Vasc Dis. 2017;10:63-5.

\section{Publisher's Note}

Springer Nature remains neutral with regard to jurisdictional claims in published maps and institutional affiliations.
Ready to submit your research? Choose BMC and benefit from:

- fast, convenient online submission

- thorough peer review by experienced researchers in your field

- rapid publication on acceptance

- support for research data, including large and complex data types

- gold Open Access which fosters wider collaboration and increased citations

- maximum visibility for your research: over $100 \mathrm{M}$ website views per year

At $\mathrm{BMC}$, research is always in progress.

Learn more biomedcentral.com/submissions 